\title{
ELECTROMAGNETIC MASS SHIFTS, EQUAL-TIME COMMUTATORS, AND JOST-LEHMANN REPRESENTATION
}

David G. Boulware*

Physics Department, University of Washington, Seattle, Washington

and

Stanley Deser $\dagger$

Physics Department, Brandeis University, Waltham, Massachusetts (Received 24 April 1968)

\begin{abstract}
Electromagnetic mass shifts, equal-time commutators (ETC), and their relation are analyzed, using the Jost-Lehmann representation for the forward Compton amplitude. The divergences in $\delta m$ are determined by Lorentz scalars associated with the ETC (when these exist). These results are illustrated by models with currents bilinear and linear in canonical fields. Existence of the ETC implies that the Jost-Lehmann representation is unsubtracted.
\end{abstract}

We analyze the general connection between asymptotic behavior of the forward Compton amplitude $\mathfrak{T l}_{\mu \lambda}\left(k^{2}, \nu\right)$ and equal-time electromagnetic (em) current commutators (ETC). When the invariant amplitudes satisfy unsubtracted $\nu^{2}$ relations and behave as $k^{-2}$ or $k^{-4}$, Bjorken has shown that the limit $k_{0} \rightarrow \infty\left(\overrightarrow{\mathrm{k}}\right.$ fixed) determines the $k^{2} \rightarrow \infty$ behavior. Since this limit also identifies the appropriate ETC, the latter then determine the quadratic and logarithmic divergences in mass shifts implied by the asymptotic $k^{-2}$ and $k^{-4}$ dependence. The precise $\delta m$-ETC relation will be given first when the Bjorken assumptions hold and will be applied to models of current interest. We then establish and use the Jost-Lehmann ${ }^{2}$ representation for $\mathfrak{M} \mu \lambda$ to study the relation under more general circumstances, and to find the necessary conditions for the ETC to exist at all.

The forward, spin-averaged, Compton amplitude is

$$
\begin{aligned}
\Re_{\mu \lambda}\left(k^{2}, \nu\right) & \equiv i \int d^{4} x e^{-i k x}\left\langle p\left|T *\left[j_{\mu}(x) j_{\lambda}(0)\right]\right| p\right\rangle, \\
& =\left(g_{\mu \lambda} k^{2}-k_{\mu}{ }^{k}\right) \mathfrak{H}_{1}\left(k^{2}, \nu\right)-m^{-2}\left[k^{2} p_{\mu} p_{\lambda}-p k\left(p_{\mu}{ }^{k}{ }_{\lambda}+p_{\lambda}{ }^{k}{ }_{\mu}\right)+g_{\mu \lambda}(p k)^{2}\right] \Re \pi_{2}\left(k^{2}, \nu\right),
\end{aligned}
$$

where $i T^{*}\left(j^{\mu} j^{\nu}\right)$ is the conserved covariant current correlation function with disconnected graphs re-

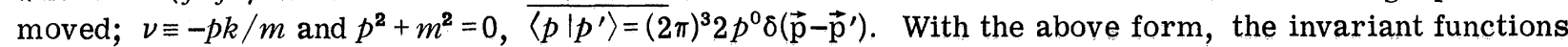
$\mathfrak{M}_{i}$ are free of kinematic singularities. The $O(\alpha)$ mass-shift is determined by the trace $\mathfrak{M}_{\mu} \mu$ (gauge invariance follows from the conservation condition):

$$
\delta m^{2}=-3 i e^{2} \int d^{4} k(2 \pi)^{-4}\left(k^{2}-i \epsilon\right)^{-1}\left\{k^{2} \mathfrak{M}_{1}+\frac{1}{3}\left[k^{2}-2(p k)^{2}\right] \mathbb{M}_{2}\right\} .
$$

Internal indices are suppressed, mass splits being given by appropriate isospin combinations of the

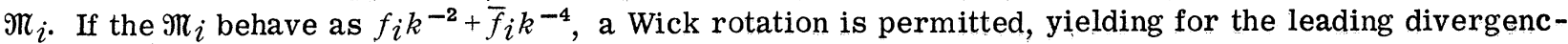
es $\delta m \sim\left(\int d k^{2}\right)\left(f_{1}+\frac{1}{2} f_{2}\right), \int k^{-2} d k^{2}\left(\bar{f}_{1}+\frac{1}{2} \bar{f}_{2}\right)$, respectively. These combinations are related to (but not identical with) the matrix elements of $C_{i} \equiv\left[j_{0}(\overrightarrow{\mathrm{r}}), j_{i}(0)\right]=C_{00 i k} \partial_{k} \delta^{3}(\overrightarrow{\mathrm{r}})$ and $C_{i k} \equiv\left[\partial_{0} j_{i}(\overrightarrow{\mathrm{r}})-\partial_{i} j_{0}(\overrightarrow{\mathrm{r}}), j_{k}(0)\right]$ $=\bar{C}_{00 i k} \delta^{3}(\overrightarrow{\mathrm{r}})$. The coefficients of $\partial_{k} \delta(\overrightarrow{\mathrm{r}})$ and $\delta(\overrightarrow{\mathrm{r}})$ are, as anticipated by our notation, components of fourth-rank tensors ${ }^{3} C_{\mu \nu \lambda \sigma}, \bar{C}_{\mu \nu \lambda \sigma}$, as is seen from the kinematic tensor form of the $k_{0} \rightarrow \infty$ limit of $\mathfrak{N}_{0 i}$,

$$
\mathfrak{N}_{0 i} \sim\left[\delta_{i}^{j} f_{1}+m^{-2}\left(\delta_{i}^{j} p^{0} p^{0}-p_{i} p^{j}\right) f_{2}\right] k_{j},
$$

and likewise for the $\bar{C}$. Thus, effectively,

$$
C_{\mu \nu \lambda \sigma}=-g_{\mu \nu} g_{\lambda \sigma} f_{1}+m^{-2}\left(p_{\mu} p_{\nu} g_{\lambda \sigma}+p_{\lambda} p_{\sigma} g_{\mu \nu}\right) f_{2}+m^{-2} p_{\mu} p_{\nu} g_{\lambda \sigma} f_{1}^{(1)} \text {. }
$$

Terms of the $f_{1}^{(1)}$ form are clearly not of the kinematically required type, but can occur, for example, in $\mathrm{SU}(2) \otimes \mathrm{SU}(2)$ algebra of fields. Terms of the form $p^{\mu} p^{\nu} p_{p}{ }_{p} \sigma$ do not arise in simple models, and have been omitted, as have also possible structures with vanishing $(00 i j)$ components because 
these do not contribute to $\delta m$ divergences; the latter depend on the combination $\mathfrak{M}_{1}+\frac{1}{2} \mathfrak{M}_{2}+\frac{1}{4} \mathfrak{M}_{1}{ }_{1}^{(1)}$, which may be shown to be the matrix element of the Lorentz scalar $S \equiv(36)^{-1}\left[C \mu \nu_{\mu \nu}-\left(\frac{5}{2}\right) C^{\mu} \mu^{\nu}{ }_{\nu}\right]$. Clearly $S$ and $C_{00 i k}$ (or even $C_{00 i i}$ ) are quite different quantities and it is only $S$ which is relevant to $\delta m^{2}$.

The operative ETC are entirely model dependent for em currents. It is therefore instructive to illustrate these results with some explicit models: (1) $j^{\mu}$ bilinear in boson (spin-1 or -0) fields, (2) $j^{\mu}$ bilinear in spin $-\frac{1}{2}$ field only, and (3) $j^{\mu}$ linear in a canonical field.

In (1), $S$ has the form

$$
S=:\left[2 \varphi^{\dagger} \varphi+\frac{3}{2} B{ }_{\mu}^{\dagger} B^{\mu}-\left(6 m^{2}\right)^{-1} G_{\mu \nu}^{\dagger} G^{\mu \nu}\right]:
$$

where $\varphi, B_{\mu}, G_{\mu \nu}$ are the charged scalar and vector fields and $G_{\mu \nu}$ the spin-1 field strength. The diagonal element will not, in general, vanish and there are quadratic divergences. For (2), even assuming that $C_{00} i k$ is a $c$-number (and this depends also on the charged fermion's other interactions), there will in general be a logarithmic divergence from the operator $\bar{S}$.

In (3), two classes of electromagnetic couplings are, in some cases, more convergent than the bilinear models. In the first, the current is proportional to a canonical field, and its curl is the conjugate momentum. Then both $\left[j_{0}, j_{i}\right]$ and $\left[\partial_{0} j_{i}-\partial_{i} j_{0}, j_{k}\right]$ are $c$-numbers and there are no divergences. In one version of this class ${ }^{4}$ the current is proportional to the $\rho^{0}$ field, so that either there is a Yang-Mills coupling (analyzed below) or there must be additional terms in the current corresponding to the $\rho^{ \pm}$, in which case these bilinear boson terms yield a quadratic divergence. In the second version ${ }^{5}$ the canonical field is a new entity, coupled to what is normally called the electromagnetic current; the mass shift is then finite, at least to $O(\alpha)$. In the second class, a Yang-Mills coupling is introduced and

$$
\left[\partial^{0} j^{i}-\partial^{i} j^{0}, j^{k}\right]=i\left[\lambda \delta^{i k}+\lambda^{\prime} \varphi_{b}{ }^{i}(0) c_{b d a}{ }^{c} a d c^{\varphi}{ }_{c}^{k}(0)\right] \delta \delta^{3}(\overrightarrow{\mathrm{r}})
$$

is no longer a $c$ number. Here the structure-constant indices include parity, so that, in $\mathrm{SU}(2) \otimes \mathrm{SU}(2)$, both vector and axial fields enter. The logarithmic divergence is then determined by

$$
\bar{S}=\frac{1}{4} m^{2}\left\langle p\left|: V^{+}{ }_{\mu}{ }^{-}+A^{+\mu_{A}}{ }_{\mu}{ }^{-}:\right| p\right\rangle
$$

and, in particular, the $(I=2)$ mass splitting of a massive, rather than Goldstone, pion is logarithmically divergent. ${ }^{6}$

To investigate the general behavior of $\mathfrak{T} \mu \lambda$, we use the Jost-Lehmann representation. ${ }^{2}$ While the latter was formulated for the causal commutator, the spectral conditions in this problem imply disjoint frequency ranges in the two orderings of the commutator, and so permit one to write it for the unordered and $T^{*}$ products as well. The invariant functions then read, in the rest frame of $p^{\mu}$,

$$
\mathfrak{N}_{i}=\int d s d^{3} u \Psi_{i}(s,|\overrightarrow{\mathrm{u}}|)\left[s+(\overrightarrow{\mathrm{u}}-\overrightarrow{\mathrm{k}})^{2}-k_{0}{ }^{2}-i \epsilon\right]^{-1},
$$

where crossing and rotational invariance have been used to eliminate terms odd in $k_{0}$ and angular dependence in $\Psi_{i}$. The amplitude $\mathfrak{M}_{\mu \lambda}$, in an arbitrary frame, is then of the kinematical form exhibited in Eq. (1). Subtractions may in general be required in the $\mathfrak{M}_{i}$. However, in that case, we shall see later that the corresponding ETC are not defined. We may therefore deal with unsubtracted $\mathfrak{M T}_{i}$ in discussing the $\delta m$-ETC relation; then the leading divergence is quadratic, the coefficient of $\int d k^{2}$ being proportional to $\int d s d^{3} u\left(\Psi_{1}+\frac{1}{2} \Psi_{2}\right)$, which is also the expectation value $\langle p|S| p\rangle$ of the ETC when expressed in terms of the $\Psi_{i}$. Similarly, if the quadratic divergences vanish then the logarithmic divergence is determined by $\int d s d^{3} u(-s)\left(\Psi_{1}+\frac{1}{2} \Psi_{2}\right)$ which is again $\langle p|\bar{S}| p\rangle$, so the commutators-when they exist-still determine the leading divergences.

The mass shifts can be calculated in terms of the Jost-Lehmann forms whether or not the ETC exist; however, the question of a relation between the two can only be meaningful if the latter exist as distributions in $\overrightarrow{\mathrm{r}}$. The Jost-Lehmann representation may also be used to find the necessary existence criteria. The conditions are obtained by considering the unordered products $F^{\mu \nu}(x-i \eta) \equiv\langle p| j \mu(x-i \eta)$ $\times j \nu(0)|p\rangle$ as analytic functions in the forward cone. Then the equal-time commutator is defined as the 
limit

$$
\lim _{\eta \rightarrow 0}\left[F^{\mu \nu}(x-i \eta)-F^{\nu \mu}(-x-i \eta)\right]_{x^{0}=0} \equiv\left\langle p\left|\left[j^{\mu}(\overrightarrow{\mathrm{r}}), j^{\nu}(0)\right]\right| p\right\rangle .
$$

The requirement that the limit exist as a distribution in $\vec{r}$ imposes conditions on the Jost-Lehmann spectral functions. These are most conveniently presented as conditions on the moments $\psi_{i}^{(n)}(s)$ $\equiv \int d^{3} u u^{2 n} \Psi_{i}(s, u)$ which are well-defined by virtue of the compact support in $\overrightarrow{\mathrm{u}}$. Explicit calculation of the small- $x^{\mu}$ behavior of $F^{\mu \nu}$ allows one to show that the equal-time commutator can exist, in all Lorentz frames, only if the large-s behavior of $\psi_{i}{ }^{(n)}(s)$ is $\psi_{i}{ }^{(n)}(s) \sim P_{i}{ }^{(n)}(s)+\Delta \psi_{i}(n)(s)$, with $P_{i}(n)(s)$ an arbitrary polynomial in $s$ and $\int_{0}^{\infty} d s \Delta \psi_{i}(0)(s)<\infty, s-n+1 \Delta \psi_{i}(n)(s) \rightarrow 0$ for $n>0$. If $P_{i}(n)(s)$ is of higher order than $s^{n-1}$, the coefficients of the higher powers must satisfy sets of linear equations. These conditions, in addition to guaranteeing the existence of the commutator, also guarantee that the commutator transforms under Lorentz transformations as a tensor-of arbitrarily high rank. The coefficients of the $s^{n-1}$ term and $\int_{0}^{\infty} d s \psi_{i}{ }^{(0)}(s)$ determine the commutator and, if there are contributions of $\psi_{i}(n)$ to the commutator for $n \leqslant N$, the commutator transforms as a $(2 N)$ th-rank tensor, in addition to the fourth-rank transformation properties dictated by the kinematical factors. The conditions also imply that the Jost-Lehmann representation does not require subtractions, hence the existence of the equal-time commutators implies that the Jost-Lehmann representation is unsubtracted.

The equal-time commutator can, in this case, still be identified as the coefficient of $1 / k^{0}$ as $k^{0} \rightarrow \infty$; there is an additional polynomial in $k^{0}$, of arbitrary order, in $\mathfrak{N}^{\mu \nu}$. However, the asymptotic behavior of the $\mathfrak{T}_{i}$ is, in general, no longer $1 / k^{2}$; there may be arbitrary terms of $O\left(k^{2 n}\right)$ for $n \geqslant 0$ and, further, the terms of $O\left(1 / k^{2}\right)$ will include forms such as $\left(1 / k^{2}\right)\left(\nu^{2} / k^{2}\right)^{n}$ which are responsible for the higher rank tensor structure discussed above.

The mass shift can now be calculated in terms of the asymptotic behavior of the $\mathfrak{M T}_{i}$. If the amplitudes are not of $O\left(1 / k^{2}\right)$, there are, in general, terms more than quadratically divergent even when ETC exist. These divergences do not even have a well-defined coefficient-different methods of evaluation give different weightings for the various terms of the same order. When the mass shift is no more than quadratically divergent, the amplitude $m_{i}$ is of the form

$$
\sum_{n} \lambda_{i}^{(n)_{k}-2}\left(-\nu^{2} / k^{2}\right)^{n}
$$

The contribution to the $\left[j^{0}, j^{k}\right]$ commutator is the limit $k_{0} \rightarrow \infty$, i.e.,

$$
i \partial_{l} \delta^{3}(\overrightarrow{\mathrm{r}})\left\{\delta^{l k} \sum_{n}{ }^{\lambda}{ }^{(n)}\left(p^{0} / m\right)^{2 n}+\left(\delta^{l k} p^{0} p^{0}-p^{l} p^{k}\right)\left(m^{-2}\right) \sum_{n} \lambda_{2}^{(n)}\left(p^{0} / m\right)^{2 n}\right\}
$$

where the $\lambda_{i}{ }^{(n)}$ are linear combinations of the coefficients of $\psi_{i}(r)(s)$ for $r \geqslant n$. On the other hand, the contribution to the mass shift is

$$
\begin{aligned}
\delta m^{2} & =-3 i e^{2} \int d^{4} k(2 \pi)^{-4}\left\{\sum_{n} \lambda_{1}{ }^{(n)_{k}-2}\left(-\nu^{2} / k^{2}\right)^{n}+\frac{1}{3}\left(1-2 \nu^{2} / k^{2}\right) \sum_{n} \lambda_{2}{ }^{(n)_{k}-2}\left(-\nu^{2} / k^{2}\right)^{n}\right\} \\
& =3 e^{2}(4 \pi)^{-2} \int^{\infty} d k^{2}\left\{\sum_{n} \lambda_{1}{ }^{(n)}\left\langle(\hat{p} \cdot \hat{k})^{2 n}\right\rangle+\frac{1}{3} \sum_{n} \lambda_{2}{ }^{(n)}\left\langle(\hat{p} \cdot \hat{k})^{2 n}-2(\hat{p} \cdot \hat{k})^{2 n+2}\right\rangle\right\},
\end{aligned}
$$

where \langle\rangle denotes an angular average in four dimensions,

$$
\left\langle(\hat{p} \cdot \hat{k})^{2 n}\right\rangle=\Gamma\left(n+\frac{1}{2}\right)\left[(n+1) ! \Gamma\left(\frac{1}{2}\right)\right]^{-1} \text {. }
$$

Comparison of these two formulas then yields the relation between $\delta m$ and the ETC in the most general case where the latter exist, and $\delta m$ is quadratic. The case $n=0$ reduces, of course, to the form $\left(f_{1}+\frac{1}{2} f_{2}\right)$ derived earlier. The logarithmic divergences become interesting if the above quadratic ones are absent. In that case, a completely analogous treatment ${ }^{8}$ can be carried through to link the parameters $\left\langle p\left|\left[\partial_{0} j_{i}-\partial_{i} j_{0}, j_{k}\right]\right| p\right\rangle$ to the logarithmic divergence coefficient.

We have seen that the divergent parts of the mass shift constitute a probe of the small-distance behavior of a theory through the highly model-dependent equal-time commutators which determine it. Conversely, knowledge of the latter in any model provides a statement of the modifications it will pro- 
duce in radiative corrections. Detailed statements of the conditions under which the ETC exist have been provided, as have precise statements of the relation between the latter and the divergences. A more complete derivation will be given elsewhere.

One of us (D.G.B.) thanks Brandeis University for hospitality while part of this work was performed.

*Work supported by the U. S. Atomic Energy Commission under Contract No. RLO 1388B.

†Work supported by the U. S. Air Force, Office of Aerospace Research, under Office of Scientific Research Grant No. 368-67.

${ }^{1}$ J. D. Bjorken, Phys. Rev. 148, 1467 (1966).

${ }^{2}$ R. Jost and H. Lehmann, Nuovo Cimento $\underline{5}, 1598$ (1957).

${ }^{3} \mathrm{It}$ is possible, in particular models, for the $(00)$ indices to be degenerate, with $C^{\mu \nu \lambda \sigma} \sim g^{\mu \nu} \widetilde{C}^{\lambda \sigma}$. In general,

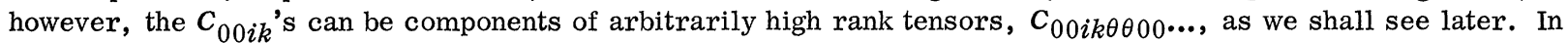
the present context we restrict ourselves to foruth-rank structure.

${ }^{4}$ T. D. Lee, S. Weinberg, and B. Zumino, Phys. Rev. Letters 18, 1029 (1967).

${ }^{5}$ T. D. Lee, to be published.

${ }^{6}$ This result was established independently by the authors [S. Deser, in Lectures of the Summer Institute of the Niels Bohr Institute, Copenhagen, Denmark, 1967 (to be published)]; M. Halpern and G. Segré, Phys. Rev. Letters 19, 611, 1000(E) (1967); and G. C. Wick and B. Zumino, Phys. Letters 25B, 479 (1967).

${ }^{7}$ These terms are also related to ETC, namely, to the higher-order derivatives of the $\delta$ function which may occur in the ETC. Throughout, when referring to ETC, we mean the lowest-order derivative term of the commutator which is allowed to occur, e.g., $\left[j^{0}, \vec{j}\right] \sim \nabla \delta(\overrightarrow{\mathrm{r}})$ and $\left[\partial 0 j^{i}-\partial i_{j} 0, j l\right] \sim \delta(\overrightarrow{\mathrm{r}})$.

${ }^{8}$ The actual calculations in this case require generalization of the conditions given in the quadratic case for the asymptotic behavior of the $\psi_{i}^{(n)}(s)$. The relevant behavior of the $\mathfrak{T}_{i}$ is $k^{-4}\left(-\nu^{2} / k^{2}\right)^{n}$ in this case. It is amusing that in some of our models, including algebra of fields (with Yang-Mills ETC), $\mathfrak{T T}_{1}$ has terms $\sim k^{-4}\left(\nu^{2} / k^{2}\right)$ which cancel parts of $\mathfrak{T l}_{2}$, exemplifying the possibility of nonkinematical structures of the type we have discussed.

\title{
MISUSES OF THE FINITE-ENERGY SUM RULES*
}

Jeffrey E. Mandula and Richard C. Slansky

California Institute of Technology, Pasadena, California

(Received 22 April 1968)

\begin{abstract}
It is shown that any model in which the scattering amplitude is given by finitely spaced trajectories of direct-channel resonances does not yield Regge asymptotic behavior in the direct channel. Several difficulties associated with the use of Regge asymptotic behavior at low values of the energy through the finite-energy sum rules are noted.
\end{abstract}

(I) The finite-energy sum rules (FESR), ${ }^{1,2}$ or generalized superconvergence relations, relate a finite integral of the imaginary part of a scattering amplitude to the crossed-channel Regge poles controlling the high-energy scattering. The FESR are easily derived from the superconvergence of $A(t, s)-\sum_{i} R_{i}(t, s)$, the amplitude minus the necessary number of leading Regge trajectories. The zeroth-moment sum rule for the scattering of equal-mass scalar particles is

$$
\frac{1}{\pi} \int^{s} d s^{\prime} \operatorname{Im} A\left(t, s^{\prime}\right) \cong \sum_{i} \beta_{i}(t) s^{\alpha_{i}(t)+1} .
$$

Certain factors have been absorbed into $\beta_{i}(t)$. The absolute value of the difference of the two sides of Eq. (1) goes to zero in the limit $s \rightarrow \infty$.
Note that for $t \leqslant 0$ the integral traverses part of the $s$-channel physical region.

In practice the FESR are used in two distinct ways. For very large $s$ they are used to determine the parameters of a representation for $\operatorname{Im} A(t, s)$ (other than the Regge representation), which is assumed to be valid over the entire range of integration. For values of $s$ currently accessible experimentally, they are used to obtain information about the Regge parameters from the low-energy data.

The most obvious and simple parametrization of $\operatorname{Im} A\left(t, s^{\prime}\right)$ when $s$ is large is a sum of directchannel poles. Such a model is particularly attractive if the Regge trajectories rise indefinitely, since one can then identify the Regge poles with the direct-channel resonances, and the 